\title{
Inhibition of TMEM45A suppresses proliferation, induces cell cycle arrest and reduces cell invasion in human ovarian cancer cells
}

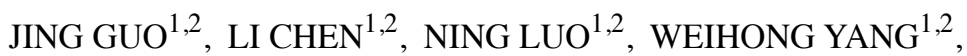 \\ XIAOYAN QU ${ }^{1,2}$ and ZHONGPING CHENG $^{1,2}$ \\ ${ }^{1}$ Department of Gynecology and Obstetrics, Yangpu Hospital, Tongji University School of Medicine; \\ ${ }^{2}$ Institute of Gynecological Minimal Invasive Medicine, Tongji University School of Medicine, Shanghai, P.R. China
}

Received January 20, 2015; Accepted March 6, 2015

DOI: 10.3892/or.2015.3902

\begin{abstract}
The association of TMEM45A with various cancers has been recently reported. However, the biological function of TMEM45A in ovarian cancer remains unclear. The present study aimed to elucidate the role of TMEM45A in regulating the biological behavior of ovarian cancer cells. We compared the expression of TMEM45A between ovarian cancer tissues and normal tissues based on RNA-sequencing data of the ovarian cancer cohort from The Cancer Genome Atlas (TCGA) project and our real-time PCR data from 25 pairs of ovarian cancer and their matched non-cancerous tissue samples. The expression of TMEM45A was then suppressed in two ovarian cancer cell lines, HO-8910 and A2780, by RNA interference. Cell proliferation, cell cycle distribution, adhesion and invasive ability were then detected using the Cell Counting Kit-8 assay (CCK-8), propidium iodide (PI) staining, and cell adhesion and Transwell assays, respectively. In addition, the mRNA and protein levels of transforming growth factor- $\beta$ (TGF- $\beta 1$ and TGF- $\beta 2$ ), Ras homolog family member A (RhoA) and Rho-associated kinase 2 (ROCK2) were detected with realtime PCR and western blotting, respectively. TCGA data and our real-time PCR results demonstrated the overexpression of TMEM45A in ovarian cancer. Silencing of TMEM45A significantly inhibited cell proliferation and significantly increased the cell population in the G1 phase. Moreover, knockdown of TMEM45A also inhibited cell adhesion as well as cell invasion. More importantly, suppression of TMEM45A notably downregulated the expression of TGF- $\beta 1$, TGF- $\beta 2$, RhoA and ROCK2. In conclusion, TMEM45A may function as an oncogene for ovarian cancer, and inhibition of TMEM45A may be a therapeutic strategy for ovarian cancer.
\end{abstract}

Correspondence to: Dr Zhongping Cheng, Department of Gynecology and Obstetrics, Yangpu Hospital, Tongji University School of Medicine, 450 Tengyue Road, Shanghai, P.R. China E-mail: mdcheng18@263.net

Key words: TMEM45A, ovarian cancer, TGF- $\beta$, RhoA/ROCK2

\section{Introduction}

Ovarian cancer is the leading cause of mortality from gynecologic malignancies in the world (1). Ovarian cancer is often diagnosed in advanced stages, resulting in a poor survival rate (2). The 5-year survival rate of patients with advanced-stage disease is merely about $5-30 \%$ (3). Recent investigations to characterize genetic alterations in ovarian cancer have discovered extensive cytogenetic and molecular alterations in these tumors (4-9). However, only limited knowledge has been obtained regarding the basic molecular mechanisms that deregulate the growth of the ovarian epithelium and lead to the invasive and metastatic behavior of these tumors.

TMEM45A (also called DERP7, DNAPTP4 or FLJ10134) belongs to the large family of genes encoding predicted transmembrane (TMEM) proteins. Recently, there are a few studies concerning the expression and functions of TMEM45A in cancers (10-13). TMEM45A was reported as an epigenetically regulated gene in MCF-7 breast cancer cells (14). Overexpression of TMEM45A has been shown to favor chemoresistance of liver and breast cancer cells under hypoxic conditions (11). It has been reported that high expression of TMEM45A is associated with poor prognosis in patients with breast (11), bladder (12) and ovarian cancer (10). However, there remains a lack of in-depth research on the expression pattern and biological functions of THEM45A in ovarian cancers.

To investigate the role of TMEM45A in ovarian cancer, we compared its expression between ovarian cancer and normal tissues. The effects of TMEM45A silencing on the proliferation, adhesion and invasion of ovarian cancer cells were then assessed. The possible involved mechanisms were also explored. Our study provides evidence that TMEM45A is overexpressed in ovarian cancer and it may be an effective therapeutic target for this disease.

\section{Materials and methods}

Bioinformatic analysis. The Cancer Genome Atlas (TCGA) RNA-sequencing and corresponding clinical data were 
downloaded from the TCGA website https://tcga-data.nci.nih. gov/tcga/ following approval of this project by the consortium. Data from 568 ovarian cancers and 8 adjacent normal tissues were used for RNA-seq analysis. To further investigate the biological pathways involved in ovarian cancer pathogenesis through the TMEM45A pathway, we performed a gene set enrichment analysis (GSEA) (15) by using GSEA version 2.0 from the Broad Institute at MIT. The data in question were analyzed in terms of their differential enrichment in a predefined biological set of genes (representing pathways). In this study, GSEA firstly generated an ordered list of all genes according to their correlation with TMEM45A expression, and then a predefined gene set (signature of gene expression upon perturbation of certain cancer-related gene) receives an enrichment score (ES), which is a measure of statistical evidence rejecting the null hypothesis that its members are randomly distributed in the ordered list. The expression level of TMEM45A was used as a phenotype label, and 'metric for ranking genes' was set to Pearson correlation. All other basic and advanced fields were set to default. The KEGG gene sets of the biological process database (c2.KEGG.v4.0) from the Molecular Signatures Database (MSig DB) (http://www.broad. mit.edu/gsea/msigdb/index.jsp) were used for enrichment analysis.

Cancer specimens. Tumor tissues and paired non-cancerous tissues were collected from 25 patients diagnosed with epithelial ovarian serous adenocarcinoma, who were admitted to the Department of Gynecology and Obstetrics, Yangpu Hospital, Tongji University School of Medicine (Shanghai, China) between 2010 and 2013. Informed consent was obtained from all patients. This study was approved by the Ethics Committee of Yangpu Hospital, Shanghai, China.

Cell lines. OVCAR3, A2780, HO-8910, CAOV3, SK-OV-3 and HEK293T cells were obtained from the American Type Culture Collection (ATCC; Rockville, MD, USA). All culture media were supplemented with $10 \%$ fetal bovine serum (FBS), $100 \mathrm{mg} / \mathrm{ml}$ penicillin $\mathrm{G}$, and $50 \mu \mathrm{g} / \mathrm{ml}$ streptomycin (all from Invitrogen Life Technologies, Carlsbad, CA, USA). The ovarian cancer cell lines, OVCAR3, A2780 and HO-8910, were cultured in RPMI-1640, and the CAOV3, SK-OV-3 and HEK293T cells were cultured in Dulbecco's modified Eagle's medium (DMEM) (all from Invitrogen Life Technologies). All cells were maintained at $37^{\circ} \mathrm{C}$ in $5 \% \mathrm{CO}_{2}$.

RNA extraction and real-time PCR. Total RNA was extracted using TRIzol reagent (Invitrogen Life Technologies) according to the manufacturer's instructions. mRNA contained in $2 \mu \mathrm{g}$ total RNA was reverse transcribed using a cDNA synthesis kit (Thermo Fisher Scientific, Rockford, IL, USA) according to the manufacturer's instructions. Real-time PCR was performed to detect mRNA levels of the indicated genes. GAPDH served as an internal control. The primers used were: TMEM45A, 5'-ACCAAGTTGGATCATGGGGA-3' and 5'-AG CCATGCCAGTTAAAGCCA-3'; GAPDH, 5'-AATCCCA TCACCATCTTC-3' and 5'-AGGCTGTTGTCATACTTC-3'; TGF- $\beta 1,5$ '-GACTACTACGCCAAGGAGGTC-3' and 5'-GAG AGCAACACGGGTTCAG-3'; TGF- $\beta 2$, 5'-AGAGCAGAA GGCGAATGG-3' and 5'-AAAGTGCAGCAGGGACAG-3';
RhoA, 5'-TAGTCCACGGTCTGGTCTTC-3' and 5'-CTTTCC ACAGGCTCCATCAC-3'; ROCK2, 5'-CAGCAATGGTA AGCGTAAAG-3' and 5'-GTAGAGACGGAGTTTCACT ATG-3'. All reactions were conducted on an ABI 7300 realtime PCR machine (Applied Biosystems, Foster City, CA, USA) using the following cycling parameters: $95^{\circ} \mathrm{C}$ for $10 \mathrm{~min}$, followed by 40 cycles of $95^{\circ} \mathrm{C}$ for $15 \mathrm{sec}$, and $60^{\circ} \mathrm{C}$ for $45 \mathrm{sec}$. The gene expression was calculated using the $\Delta \Delta \mathrm{Ct}$ method. All data represent the average of 3 replicates.

RNA interference and construction of stable cell lines. Three shRNAs targeting position 713-733 (GAGGCCTTTATCTTC TACAAC; named TMEM45A-Ri-1), position 821-841 (GAGT TCCTTGTTCGGAACAAT; named TMEM45A-Ri-2) and position 1248-1268 (TAAGTGTACTGTTTGCATTTC; named TMEM45A-Ri-3) of human TMEM45A mRNA were cloned into a lentiviral vector (PLKO.1). A non-specific scramble shRNA sequence (CCTAAGGTTAAGTCGCCC TCG) was used as a negative control. The constructs were then transfected into HEK293T cells with lentiviral packaging vectors by using Lipofectamine 2000 (Invitrogen) according to the manufacturer's instructions. Viruses were collected $48 \mathrm{~h}$ after transfection and used to infect the A2780 and HO-8910 cells.

Western blotting. The cells were lysed with radioimmunoprecipitation assay buffer $(50 \mathrm{mmol} / 1 \mathrm{Tris}-\mathrm{HCl}, 150 \mathrm{mmol} / \mathrm{l}$ $\mathrm{NaCl}, 1 \%$ Triton $\mathrm{X}-100,0.1 \%$ SDS and $1 \%$ deoxycholic acid sodium). The lysates were quantified using the BCA protein assay kit (Thermo Fisher Scientific). The lysates with equal amounts of protein were separated on SDS-PAGE gels followed by electroblotting to nitrocellulose membranes. Western blotting was then carried out with appropriate primary and horseradish peroxidase-conjugated secondary antibodies. Membranes were developed with enhanced chemiluminescence (Bio-Rad, Richmond, CA, USA). Antibodies against TMEM45A, TGF $\beta 1$, TGF $\beta 2$, RhoA and ROCK 2 were purchased from Abcam (Cambridge, MA, USA). GAPDH antibody was from Cell Signaling Technology Inc. (Danvers, MA, USA).

Cell proliferation assay. A total of $3 \times 10^{3}$ cells/well were plated in 96-well plates before viral infection and cultured for $24 \mathrm{~h}$ in normal conditions. They were then infected with the TMEM45A-shRNA virus or control virus (NC). Cell proliferation was detected at the indicated times using CCK-8 (Dojindo Laboratories, Kumamoto, Japan) according to the manufacturer's instructions. Briefly, at the indicated time-points, CCK- 8 solution (10 $\mu 1$ in $100 \mu$ l DMEM) was added to each well and incubated for $1 \mathrm{~h}$. Optical density (OD) values at wavelength $450 \mathrm{~nm}$ were measured by a microplate reader (Bio-Rad Laboratories Inc., Hercules, CA, USA).

Cell cycle analysis. The cell cycle was evaluated by flow cytometry using propidium iodide (PI; Sigma, St. Louis, MO, USA) staining on a flow cytometer (BD Biosciences, Franklin Lakes, NJ, USA). Briefly, the cells were plated in 6-well plates before viral infection and cultured for $24 \mathrm{~h}$ under normal conditions. The cells were collected and fixed in $70 \%$ ethanol at $-20^{\circ} \mathrm{C}$ overnight $24 \mathrm{~h}$ after viral infection. The 

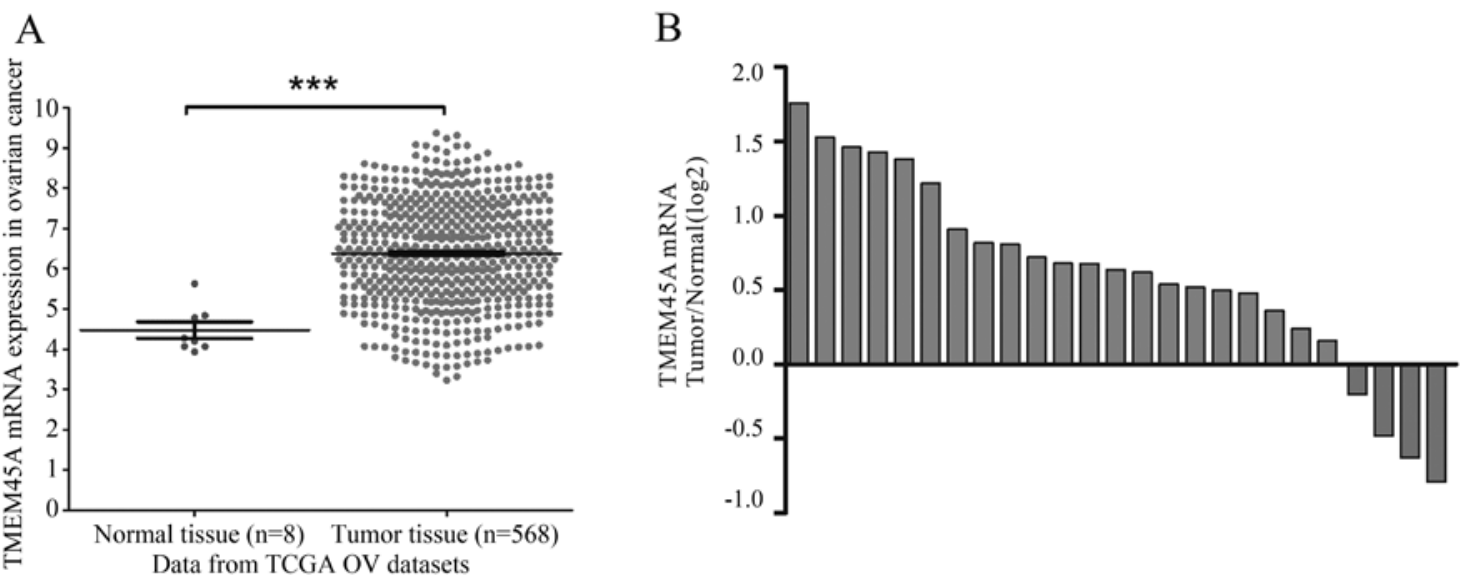

Figure 1. Overexpression of TMEM45A in ovarian cancer. (A) RNA-sequencing analysis of TMEM45A mRNA expression in ovarian cancer and normal tissues. RNA-seq analysis used data downloaded from the TCGA ovarian cancer datasets. ${ }^{* *} \mathrm{P}<0.005$. (B) The mRNA level of TMEM45A in 25 pairs of ovarian tumor and normal tissues was detected by real-time PCR. Positive $\log _{2}$ (Tumor/Normal) on the y-axis indicates increased expression of TMEM45A while negative $\log _{2}$ indicates decreased expression of TMEM45A in the tumor tissues. TMEM45A mRNA was significantly overexpressed in ovarian tumor tissues as compared with the normal tissues $(\mathrm{P}<0.001)$. TCGA, The Cancer Genome Atlas.

cells were then washed in phosphate-buffered saline (PBS) and resuspended in staining solution containing $20 \mu \mathrm{g} / \mathrm{ml} \mathrm{PI}$ and $200 \mu \mathrm{g} / \mathrm{ml}$ RNase A. Experiments were performed in triplicate and $3 \times 10^{4}$ cells were analyzed per sample. G1, S, and G2/M fractions were quantified using CellQuest software (BD Biosciences) and manual gating.

Cell adhesion assay. The adhesion assay was performed in 12 -well plates. The plates were pre-coated with $1 \mathrm{ml}$ of fibronectin $(5 \mu \mathrm{g} / \mathrm{ml})$ for $2 \mathrm{~h}$ at room temperature. The cells were infected with the indicated viral vectors $48 \mathrm{~h}$ before the assay was performed. The cells were seeded into the coated plates at a density of $10^{5}$ cells/well and allowed to adhere at $37^{\circ} \mathrm{C}$ for $1 \mathrm{~h}$. Non-adherent cells were washed off with PBS and fixed in $4 \%$ paraformaldehyde and stained with Giemsa solution. The number of adherent cells was determined as described previously (16).

In vitro invasion assay. The upper well of the Transwell (Corning Inc., Corning, NY, USA) was coated with Matrigel (BD Biosciences, San Jose, CA, USA) at $37^{\circ} \mathrm{C}$ in a $5 \% \mathrm{CO}_{2}$ incubator for $1 \mathrm{~h}$. The indicated cells were serum-starved for $24 \mathrm{~h}$, and then $500 \mu \mathrm{l}$ of cell suspension containing $10^{5}$ cells $/ \mathrm{ml}$ were placed in the upper compartment of the chamber. Culture medium supplemented with 10\% FBS (750 $\mu \mathrm{l})$ was added into the lower well of the chamber. The plates were incubated for $48 \mathrm{~h}$. At the end of the incubation, the cells on the upper surface of the filter were completely removed by wiping with a cotton swab. The cells that migrated into the lower well were washed with PBS, fixed in $4 \%$ paraformaldehyde and stained by $0.2 \%$ crystal violet. The invading cells were observed under a microscope (magnification, x100). Cells were counted in the central field of triplicate membranes.

Statistical analysis. The data were analyzed using the two-tailed Student's t-test to calculate the statistical significance of difference between groups. The results are presented as the mean value $\pm \mathrm{SD}$. Statistically significant differences were defined as having a P-value $<0.05$.

\section{Results}

Overexpression of TMEM45A in ovarian cancer. To explore the expression of TMEM45A in ovarian cancer, we compared TMEM45A by analyzing high throughput RNA-sequencing data of the ovarian cancer cohort from TCGA. TMEM45A expression was significantly higher in the ovarian cancer tissues than that in the adjacent tissues of the patients, which indicated that TMEM45A may be an oncogene in ovarian cancer (Fig. 1A).

To further confirm the overexpression of TMEM45A in ovarian cancer, we performed real-time PCR analysis on 25 pairs of ovarian cancer and their matched non-cancerous tissue samples. TMEM45A was found to be overexpressed in $84 \%(21 / 25)$ of the tested ovarian cancer tissues (Fig. 1B). Statistical analysis using the Student's t-test indicated a significant difference in the mRNA level of TMEM45A between the ovarian tumor and normal tissues $(\mathrm{P}<0.001)$.

Downregulation of TMEM45A by RNA interference (RNAi) in ovarian cancer cells. The protein and mRNA levels of TMEM45A in five ovarian cancer cell lines were then detected. A high level of TMEM45A was observed in the HO-8910 and A2780 cells (Fig. 2A). For that reason, these two cells were selected for the following assays.

To explore the functions of TMEM45A in ovarian cancer, we suppressed TMEM45A levels in the HO-8910 and A2780 cells by lentiviral-mediated delivery of shRNA. In the present study, three pairs of shRNA sequences against the human TMEM45A gene were designed and a non-specific scramble shRNA sequence was used as the negative control (NC). The silencing effect of the TMEM45A-RNAi virus was confirmed in the HO-8910 (Fig. 2B) and A2780 (Fig. 2C) cells by western blotting and real-time PCR. Our results demonstrated that TMEM45A-Ri-3 was the most efficient one and was selected for the following assays.

TMEM45A knockdown inhibits cell proliferation and induces G1-phase cell cycle arrest in ovarian cancer cells. 

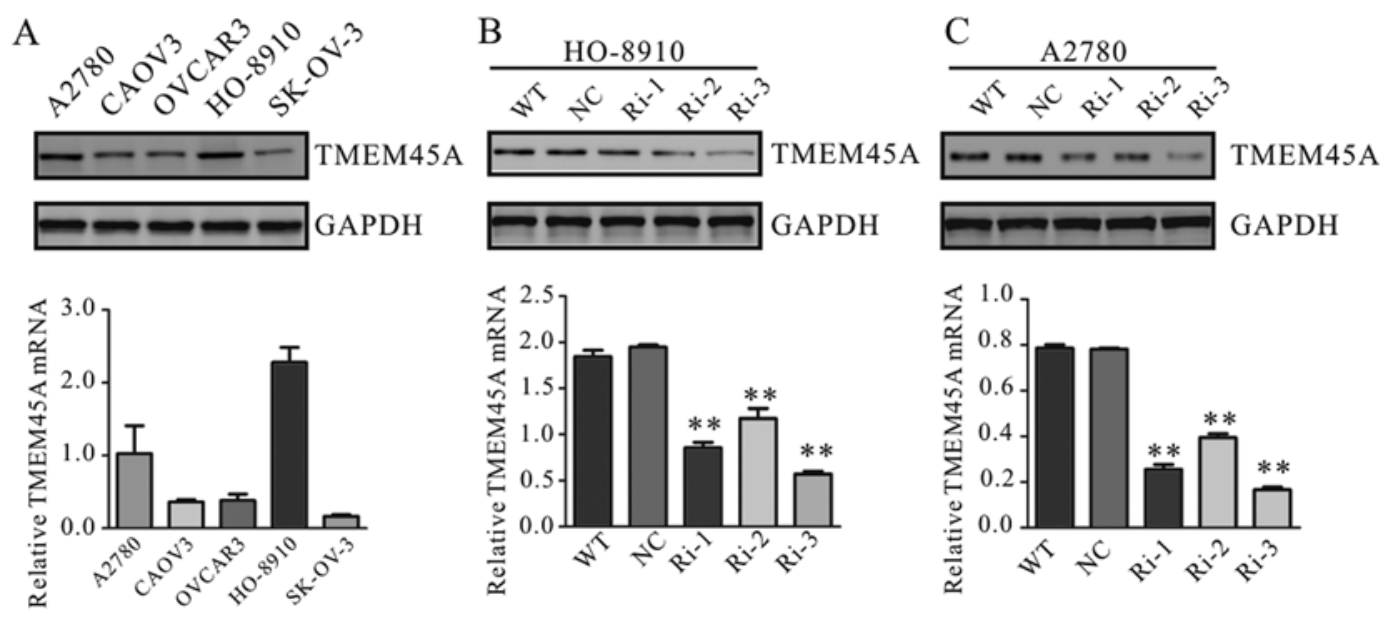

D $\mathrm{HO}-8910$
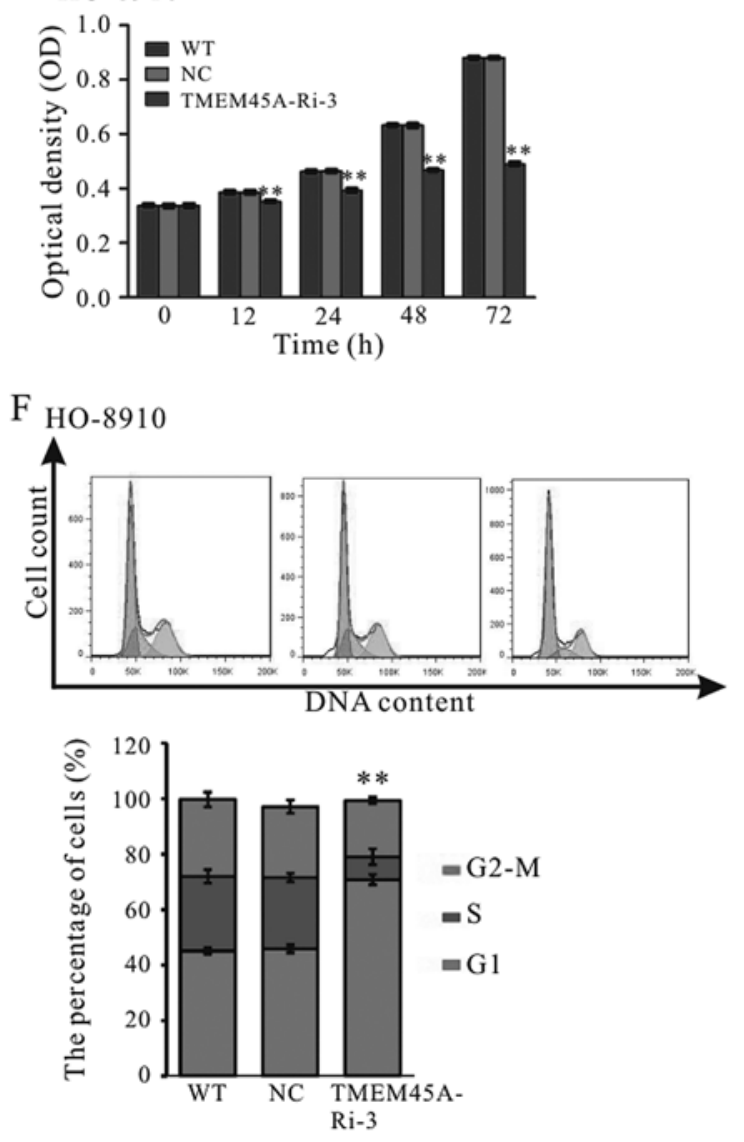

E
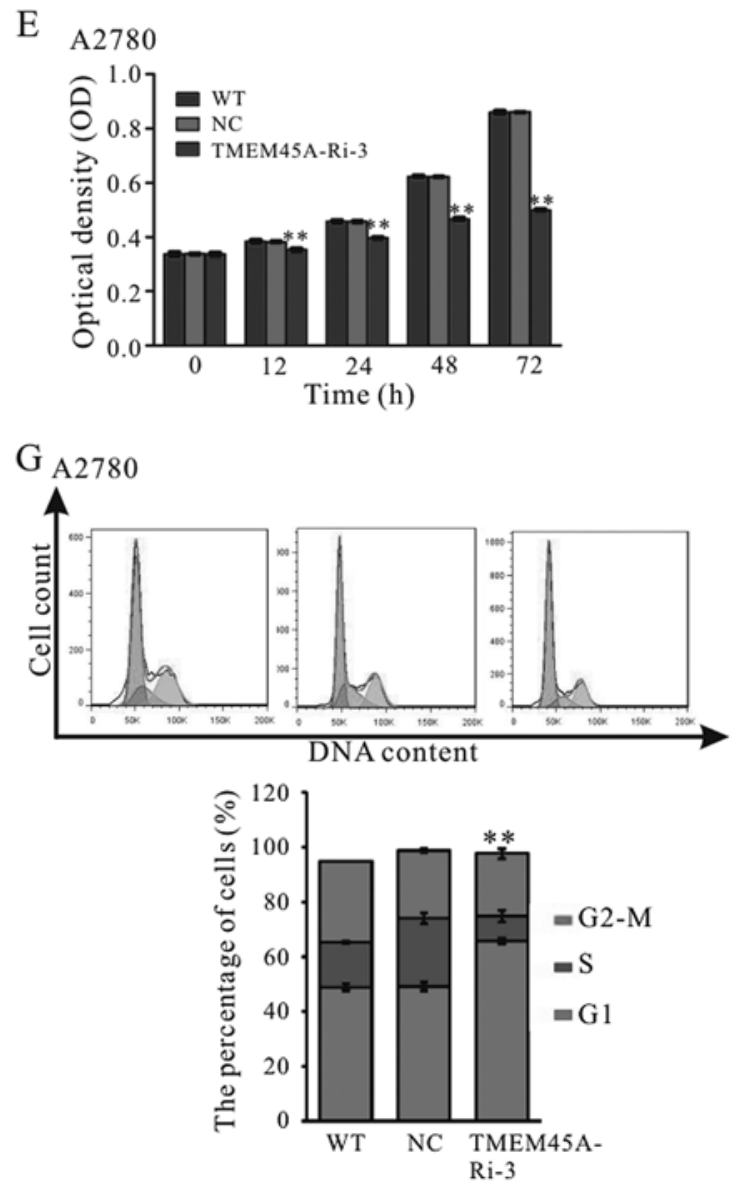

Figure 2. TMEM45A knockdown impairs cell proliferation and cell cycle progression in the HO-8910 and A2780 cells. (A) TMEM45A expression in five ovarian cancer cell lines was detected by western blotting and real-time PCR. GAPDH was used as an internal control. High expression of TMEM45A was observed in the A2780 and HO-8910 cells, which were selected for further analysis. (B and C) Western blotting (upper panel) and real-time PCR (lower panel) analyses showing the efficiency of TMEM45A knockdown in the HO-8910 and A2780 cells. NC, scrambled shRNA viral-infected cells; WT, wildtype cells; Ri-1, Ri-2 and Ri-3, TMEM45A-shRNA-1, -2 and -3 viral-infected cells. (D and E) CCK-8 assay was performed to evaluate the cell proliferation of the HO-8910 and A2780 cells. (F and G) The percentages of cells in the G1, S and G2-M phases for each sample at $48 \mathrm{~h}$ after viral infection are shown. NC, scrambled shRNA viral-infected cells; WT, wild-type cells; TMEM45A-Ri-3, TMEM45A-shRNA-3 viral-infected cells. Data are shown as the mean value $\pm \mathrm{SD}^{* *} \mathrm{P}<0.01$, vs. NC.

To investigate the role of TMEM45A downregulation on cell proliferation, we assessed the proliferation of HO-8910 and A2780 cells infected with TMEM45A-Ri-3 using the CCK-8 assay. As shown in Fig. 2D and E, cell growth was notably impaired in the TMEM45A-Ri-3 viral-infected cells (TMEM45A-Ri-3) compared to the wild-type (WT) cells and the scramble shRNA viral-infected (NC) cells. Our data indicated a role of TMEM45A in the regulation of ovarian cancer cell proliferation.

The possible effect of TMEM45A RNAi on cell cycle progression was then determined. As shown in Fig. $2 \mathrm{~F}$, a higher number of $\mathrm{HO}-8910$ cells in the G1 phase $(70.8 \pm 1.9 \%)$ was observed in cells infected with the TMEM45A shRNA virus, compared with that in the WT cells $(45.1 \pm 1.1 \%)$ and 
A

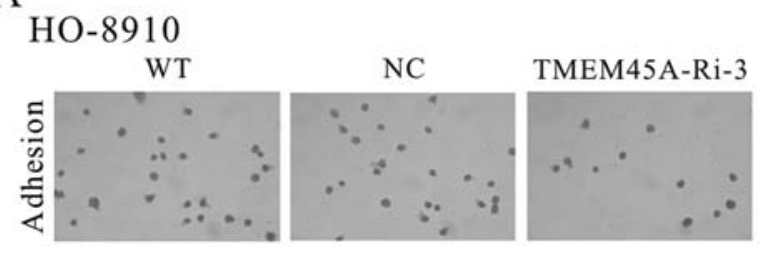

$\mathrm{B}_{\mathrm{A} 2780}$

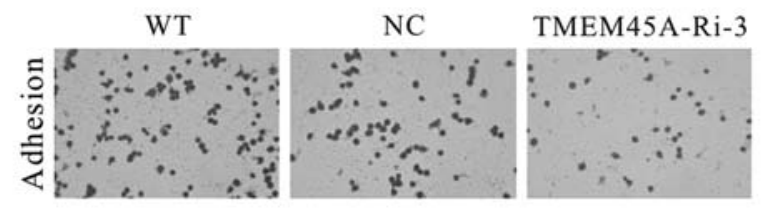

E HO- 8910

WT

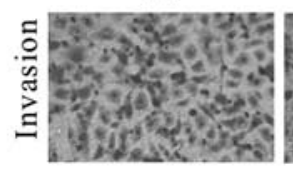

F A2780

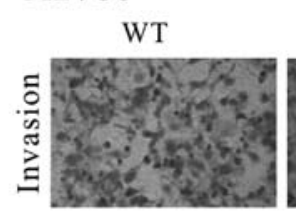

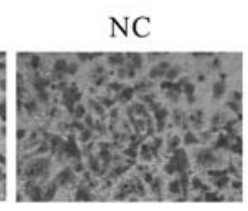

TMEM45A-Ri-3

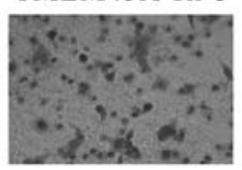

G

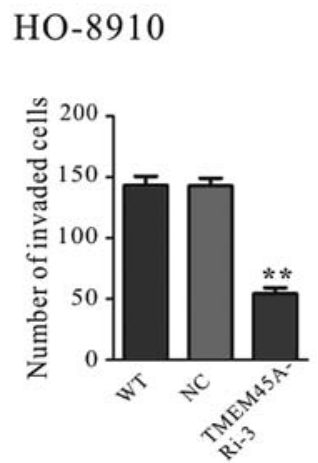

D

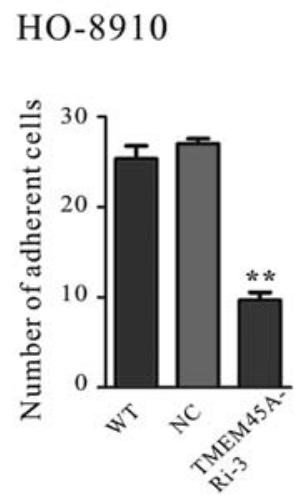

A2780

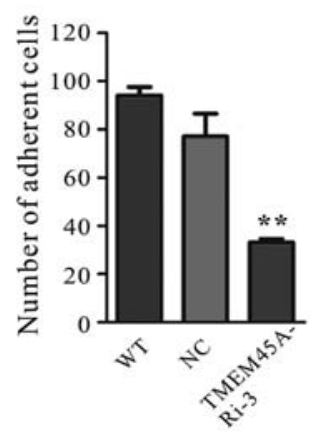

$\mathrm{H}$

\section{A2780}

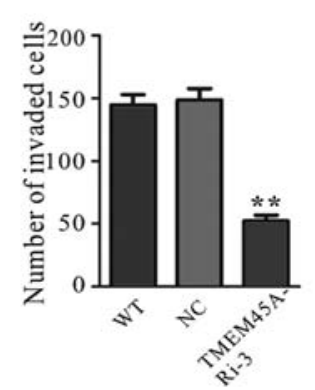

Figure 3. TMEM45A knockdown inhibits cell adhesion and invasion. (A and B) Cell adhesion assay was performed in the control and TMEM45A-knockdown cells. TMEM45A knockdown notably decreased the cell adhesion capacity of the HO-8910 and A2780 cells. Representative images of the cell adhesion assay are shown. (C and D) Quantitative results of the cell adhesion assay. (E and F) Invasion assay of control and TMEM45A-knockdown HO-8910 and A2780 cells in Matrigel-coated Transwell chambers. Cells that migrated from the upper well of the Transwell chamber into the lower well were stained, photographed and counted. Representative images of cell invasion assay are shown. (G and H) Quantitative results of the cell invasion assay. WT, wild-type cells. NC, scrambled shRNA viral-infected cells; TMEM45A-Ri-3, TMEM45A-shRNA-3 viral-infected cells. Data are shown as the mean value \pm SD. All data are representative results of three independent experiments. ${ }^{*} \mathrm{P}<0.05,{ }^{* *} \mathrm{P}<0.01$, vs. NC.

NC cells $(45.9 \pm 1.1 \%)$. Similar results were observed in the A2780 cells (Fig. 2G). These results indicated that the proliferation-promoting function of TMEM45A was most likely mediated by promoting G1/S cell cycle transition in the ovarian cancer cells.

Suppression of TMEM45A expression represses cell adhesion capacity. The effects of TMEM45A on cell adhesion capacity were evaluated by a cell adhesion assay (Fig. 3). The adherent ability of ovarian cells to fibronectin was significantly repressed by TMEM45A knockdown. The number of adherent TMEM45A-Ri-3 cells was $37 \%$ of that of the NC cells when the HO-8910 cells were used (Fig. 3A and C). Similar results were obtained for the A2780 cells (Fig. 3B and D). These data suggest that TMEM45A may regulate ovarian cancer cell adhesion.

TMEM45A knockdown inhibits the invasiveness of ovarian cancer cells. To discover whether TMEM45A affects the invasive ability of ovarian cancer cells, we performed a Matrigel-coated membrane chamber invasion assay. As shown in Fig. 3, a radical reduction in the invasive ability was observed in the TMEM45A-knockdown cells compared to the control cells. The number of invaded TMEM45A-Ri-3 cells decreased to $38 \%$ of that of the NC cells when the HO-8910 cells were used (Fig. 3E and G). Similar results were observed for the A2780 cells (Fig. 3F and H).

Identification of genes and signaling-associated biological pathways and processes by GSEA. The exact pathway that TMEM45 may regulate in ovarian cancers remains unclear. To probe the TMEM45A-associated pathways on an unbiased basis, we performed GSEA using high throughput RNA-sequencing data of the ovarian cancer cohort from TCGA. GSEA is designed to detect coordinated differences in expression of predefined sets of functionally related genes. Among all the 188 predefined 'KEGG pathway' gene sets, the TGF- $\beta$ signaling pathway and focal adhesion pathway were identified as having a significant association with higher expression of TMEM45A (Fig. 4A and B).

TMEM45A knockdown downregulates the $m R N A$ and protein levels of TGF- $\beta 1, T G F-\beta 2$, RhoA and ROCK2. To further confirm the association of TMEM45A expression and the TGF- $\beta$ signaling and focal adhesion pathways, we detected the expression of important regulators of the TGF- $\beta$ signaling 
A

KEGG TGF- $\beta$ signaling pathway

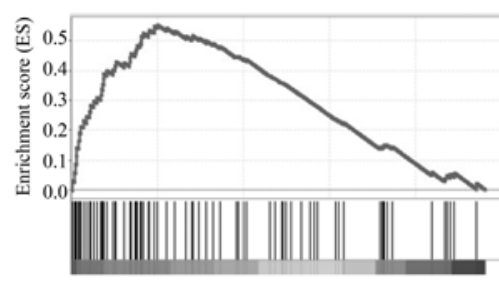

TMEM45A

TMEM45A

higher expression lower expression
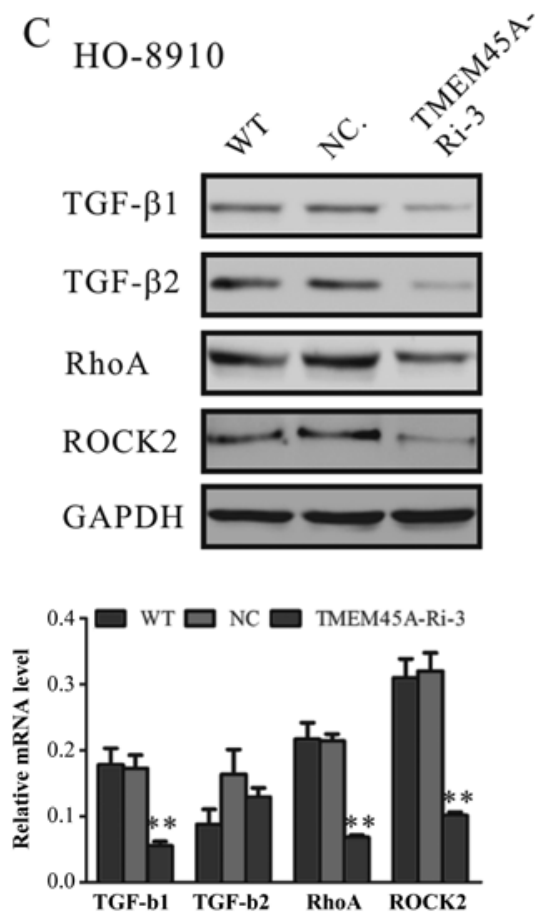

$\mathrm{B}$

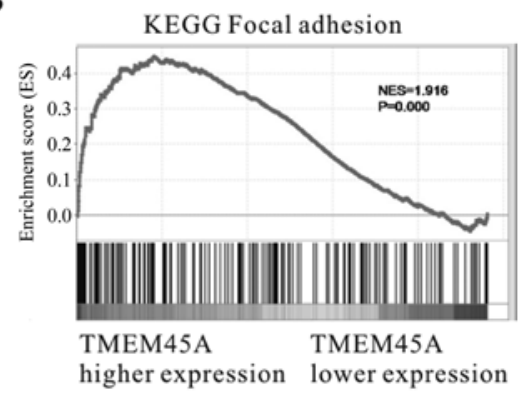

$\mathrm{D}$
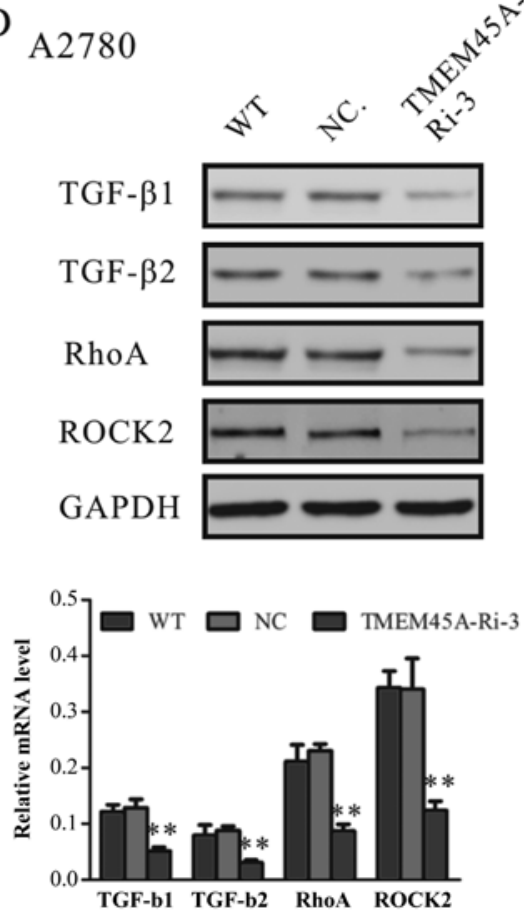

Figure 4. Gene set enrichment analysis of genes overexpressed in TMEM45A-higher expression patients relative to TMEM45A-lower expression patients from the TCGA ovarian cancer dataset. (A) KEGG TGF- $\beta$ signaling pathway and (B) focal adhesion genes were positively correlated with TMEM45A expression. Western blotting (upper panel) and real-time PCR analysis (lower panel) identified that expression of TGF- $\beta 1$, TGF- $\beta 2$, RhoA and ROCK2 was significantly decreased by TMEM45A RNAi in (C) HO-8910 and (D) A2780 cells. ${ }^{* *} \mathrm{P}<0.01$, vs NC. TCGA, The Cancer Genome Atlas; TGF- $\beta$, transforming growth factor- $\beta$; RhoA, Ras homolog family member A; ROCK2, Rho-associated kinase 2.

pathway and cell adhesion molecules in the TMEM45A-Ri-3 and NC cells. The mRNA and protein levels of TGF- $\beta 1$, TGF- $\beta 2$, RhoA and ROCK 2 were significantly decreased in both the HO-8910 (Fig. 4C) and A2780 (Fig. 4D) cells following downregulation of TMEM45A.

\section{Discussion}

Recently, the expression and functions of TMEM45A in cancers have been reported (10-13). In the present study, bioinformatic analysis using high throughput RNA-sequencing data from TCGA demonstrated a higher expression of TMEM45A in ovarian cancer compared to that in normal tissues (Fig. 1A). We then evaluated the mRNA levels of TMEM45A in 25 pairs of ovarian tumor and normal tissues by real-time PCR. We found that TMEM45A was overexpressed in $84 \%$ (21/25) of the tested ovarian cancer tissues (Fig. 1B).

To further investigate the functions of TMEM45A in ovarian cancer, we suppressed the expression of TMEM45A in two ovarian cell lines, HO-8910 and A2780, by RNA interfer- ence (Fig. 2B and C). Our data showed that suppression of TMEM45A expression markedly repressed the cell proliferation (Fig. 2D and E). Abnormal regulation of the cell cycle is frequently observed in most common malignancies, resulting in aberrant cell proliferation $(17,18)$. In the present study, silencing of TMEM45A by RNAi significantly stimulated cell cycle arrest in the G1-phase (Fig. 2F and G), which indicated that TMEM45A promoted cell proliferation by promoting G1/S cell cycle transition in ovarian cancer cells. Moreover, cell adhesion and invasion of ovarian cells were also inhibited by TMEM45A downregulation (Fig. 3). These data suggest a role of TMEM45A in ovarian cell carcinogenesis.

The exact pathway that TMEM45 may regulate in ovarian cancers remains unclear. Our GSEA data indicated that TMEM45A overexpression was positively correlated with the TGF- $\beta$ signaling pathway (Fig. $4 \mathrm{~A}$ ). TGF- $\beta$ signaling participates in a variety of cellular processes, including cell differentiation, proliferation, apoptosis, and determination of developmental fate (19). The TGF- $\beta$ signaling pathway has also been considered to promote tumor progression and 
invasion. In response to elevated TGF- $\beta$ levels, the tumor cell becomes more migratory and invasive $(20,21)$. In the present study, TMEM45A knockdown significantly decreased the expression of TGF- $\beta 1$ and TGF- $\beta 2$ (Fig. 4), which indicates an association between TMEM45A function and the regulation of TGF- $\beta$ signaling in ovarian cancer cells.

Moreover, our GSEA results indicated a positive correlation between TMEM45A overexpression and focal adhesion genes (Fig. 4B). Rho is required for the formation and maintenance of focal adhesion. It has been shown that RhoA, a member of the Rho subfamily, plays a central role in the regulation of cell survival, motility, apoptosis and invasion $(22,23)$. RhoA expression was reported to be upregulated in lung, breast, colon and ovarian cancer. The expression level of RhoA may be positively correlated with the progression of these carcinomas, suggesting that RhoA may play an important role in tumorigenesis and tumor progression (24-28). The malignant phenotype in gastric cancer (29) and breast cancer (30) cells can be reversed by the inhibition of RhoA expression. Suppression of ROCK2 expression was reported to impair anchorage-independent growth and invasion of nonsmall cell lung cancer (31). In the present study, TMEM45A knockdown markedly decreased the mRNA and protein levels of RhoA and ROCK2. The dowregulation of RhoA and ROCK2 may be associated with the impaired adhesion and invasive ability (Fig. 3) of the TMEM45A RNAi cells. To our knowledge, our data are the first to associate the functions of TMEM45A with the RhoA/ROCK2 signaling pathway.

In conclusion, our study demonstrated the overexpression of TMEM45A in ovarian cancer, which was related with cancerous transformation. Our study is the first to link TMEM45A with the regulation of TGF- $\beta$ signaling and the RhoA/ROCK signaling pathway, and thus provides useful information for targeted therapy.

\section{References}

1. Zeinoun Z, Teugels E, De Bleser PJ, Neyns B, Geerts A and De Greve J: Insufficient TGF-beta 1 production inactivates the autocrine growth suppressive circuit in human ovarian cancer cell lines. Anticancer Res 19: 413-420, 1999.

2. Jemal A, Siegel R, Ward E, Hao Y, Xu J, Murray T and Thun MJ: Cancer statistics, 2008. CA Cancer J Clin 58: 71-96, 2008.

3. Cannistra SA: Cancer of the ovary. N Engl J Med 351: 2519-2529, 2004.

4. Spentzos D, Levine DA, Ramoni MF, Joseph M, Gu X, Boyd J, Libermann TA and Cannistra SA: Gene expression signature with independent prognostic significance in epithelial ovarian cancer. J Clin Oncol 22: 4700-4710, 2004.

5. Berchuck A, Iversen ES, Lancaster JM, Pittman J, Luo J, Lee P, Murphy S, Dressman HK, Febbo PG, West M, et al: Patterns of gene expression that characterize long-term survival in advanced stage serous ovarian cancers. Clin Cancer Res 11: 3686-3696, 2005.

6. Hartmann LC, Lu KH, Linette GP, Cliby WA, Kalli KR, Gershenson D, Bast RC, Stec J, Iartchouk N, Smith DI, et al: Gene expression profiles predict early relapse in ovarian cancer after platinum-paclitaxel chemotherapy. Clin Cancer Res 11: 2149-2155, 2005

7. Spentzos D, Levine DA, Kolia S, Otu H, Boyd J, Libermann TA and Cannistra SA: Unique gene expression profile based on pathologic response in epithelial ovarian cancer. J Clin Oncol 23: 7911-7918, 2005

8. Helleman J, Jansen MP, Span PN, van Staveren IL, Massuger LF, Meijer-van Gelder ME, Sweep FC, Ewing PC, van der Burg ME, Stoter G, et al: Molecular profiling of platinum resistant ovarian cancer. Int J Cancer 118: 1963-1971, 2006.
9. Dressman HK, Berchuck A, Chan G, Zhai J, Bild A, Sayer R, Cragun J, Clarke J, Whitaker RS, Li L, et al: An integrated genomic-based approach to individualized treatment of patients with advanced-stage ovarian cancer. J Clin Oncol 25: 517-525, 2007.

10. Crijns AP, Fehrmann RS, de Jong S, Gerbens F, Meersma GJ, Klip HG, Hollema H, Hofstra RM, te Meerman GJ, de Vries EG, et al: Survival-related profile, pathways, and transcription factors in ovarian cancer. PLoS Med 6: e24, 2009.

11. Flamant L, Roegiers E, Pierre M, Hayez A, Sterpin C, De Backer O, Arnould T, Poumay Y and Michiels C: TMEM45A is essential for hypoxia-induced chemoresistance in breast and liver cancer cells. BMC Cancer 12: 391, 2012.

12. Urquidi V, Goodison S, Cai Y, Sun Y and Rosser CJ: A candidate molecular biomarker panel for the detection of bladder cancer. Cancer Epidemiol Biomarkers Prev 21: 2149-2158, 2012.

13. Jinawath N, Chamgramol Y, Furukawa Y, Obama K, Tsunoda T, Sripa B, Pairojkul C and Nakamura Y: Comparison of gene expression profiles between Opisthorchis viverrini and non-Opisthorchis viverrini associated human intrahepatic cholangiocarcinoma. Hepatology 44: 1025-1038, 2006.

14. Hsieh TC and Wu JM: Differential control of growth, cell cycle progression, and gene expression in human estrogen receptor positive MCF-7 breast cancer cells by extracts derived from polysaccharopeptide I'm-Yunity and Danshen and their combination. Int J Oncol 29: 1215-1222, 2006.

15. Subramanian A, Kuehn H, Gould J, Tamayo P and Mesirov JP: GSEA-P: a desktop application for gene set enrichment analysis. Bioinformatics 23: 3251-3253, 2007.

16. Silletti S, Paku S and Raz A: Autocrine motility factor and the extracellular matrix. I. coordinate regulation of melanoma cell adhesion, spreading and migration involves focal contact reorganization. Int J Cancer 76: 120-128, 1998.

17. Evan GI and Vousden KH: Proliferation, cell cycle and apoptosis in cancer. Nature 411: 342-348, 2001.

18. Molinari M: Cell cycle checkpoints and their inactivation in human cancer. Cell Prolif 33: 261-274, 2000.

19. Shi Y and Massagué J: Mechanisms of TGF-beta signaling from cell membrane to the nucleus. Cell 113: 685-700, 2003.

20. Wakefield LM and Roberts AB: TGF-beta signaling: Positive and negative effects on tumorigenesis. Curr Opin Genet Dev 12: 22-29, 2002.

21. Vergara D, Merlot B, Lucot JP, Collinet P, Vinatier D, Fournier I and Salzet M: Epithelial-mesenchymal transition in ovarian cancer. Cancer Lett 291: 59-66, 2010.

22. Schmitz AA, Govek EE, Böttner B and Van Aelst L: Rho GTPases: signaling, migration, and invasion. Exp Cell Res 261: $1-12,2000$.

23. Aznar S and Lacal JC: Rho signals to cell growth and apoptosis. Cancer Lett 165: 1-10, 2001

24. Fritz G, Just I and Kaina B: Rho GTPases are over-expressed in human tumors. Int J Cancer 81: 682-687, 1999.

25. Abraham MT, Kuriakose MA, Sacks PG, Yee H, Chiriboga L, Bearer EL and Delacure MD: Motility-related proteins as markers for head and neck squamous cell cancer. Laryngoscope 111: 1285-1289, 2001

26. Kamai T, Arai K, Tsujii T, Honda M and Yoshida K: Overexpression of RhoA mRNA is associated with advanced stage in testicular germ cell tumour. BJU Int 87: 227-231, 2001.

27. Horiuchi A, Imai T, Wang C, Ohira S, Feng Y, Nikaido T and Konishi I: Up-regulation of small GTPases, RhoA and RhoC, is associated with tumor progression in ovarian carcinoma. Lab Inves 83: 861-870, 2003.

28. Kamai T, Tsujii T, Arai K, Takagi K, Asami H, Ito $\mathrm{Y}$ and Oshima H: Significant association of Rho/ROCK pathway with invasion and metastasis of bladder cancer. Clin Cancer Res 9: 2632-2641, 2003.

29. Liu N, Bi F, Pan Y, Sun L, Xue Y, Shi Y, Yao X, Zheng Y and Fan D: Reversal of the malignant phenotype of gastric cancer cells by inhibition of RhoA expression and activity. Clin Cancer Res 10: 6239-6247, 2004.

30. Pille JY, Denoyelle C, Varet J, Bertrand JR, Soria J, Opolon P, Lu H, Pritchard LL, Vannier JP, Malvy C, et al: Anti-RhoA and anti-RhoC siRNAs inhibit the proliferation and invasiveness of MDA-MB-231 breast cancer cells in vitro and in vivo. Mol Ther 11: 267-274, 2005.

31. Vigil D, Kim TY, Plachco A, Garton AJ, Castaldo L, Pachter JA, Dong H, Chen X, Tokar B, Campbell SL, et al: ROCK1 and ROCK 2 are required for non-small cell lung cancer anchorage-independent growth and invasion. Cancer Res 72: 5338-5347, 2012. 